\title{
A interação entre prescritores, dispensadores e pacientes: informação compartilhada como possível benefício terapêutico
}

\author{
Interaction between prescribers, dispensers, \\ and patients: shared information as a possible \\ therapeutic benefit
}

Vera Lúcia Edais Pepe 1,2

Claudia G. S. Osorio de Castro 2,3

\footnotetext{
1 Departamento de Administração e

Planejamento em Saúde, Escola Nacional de Saúde Pública, Fundação Oswaldo Cruz. Rua Leopoldo Bulhões 1480, sala 704, Rio de Janeiro, RJ 21041-210, Brasil. verapepe@ensp.fiocruz.br 2 Núcleo de Assistência Farmacêutica, Escola Nacional de Saúde Pública, Fundação Oswaldo Cruz. Rua Leopoldo Bulhões 1480 sala 406, Rio de Janeiro, RJ 21041-210, Brasil. 3 Serviço de Farmácia, Instituto Fernandes Figueira. Av. Rui Barbosa 716, Rio de Janeiro, RJ 22250-020, Brasil. claudia@iff.fiocruz.br
}

\begin{abstract}
The article presents a critical view of the interaction between prescribers, dispensers, and patients, considering information one of the key issues in enhancing the qualitative aspects involved in this complex relationship. It describes the acquisition of information by health professionals, possible sources of this information, and the process involved in transforming it into knowledge. Briefly discussed are the physi cian's and pharmaci st's roles, the pati ent's expectations as recipient, and consequences of pertinent health interventions.

Key words Drug Prescriptions; Pharmaceutical Services; Drug Utilization; Drug Information; Professional-Patient Relations
\end{abstract}

Resumo O texto apresenta uma visão crítica da interação entre prescritores, dispensadores e paci entes, vislumbrando, na informação, um dos fatores-chave para o desenvolvimento qualitativo dessa complexa relação. Descreve, além do aporte de informações por parte desses profissi onais de saúde, as fontes às quais possi vel mente terão acesso e al guns fatores envolvidos na transformação da informação em conheci mento. Relata os papéis dos profissionais que exercem o ato da prescrição e da dispensação, as expectativas do paciente como alvo dos serviços prestados eas conseqüênci as das ações de saú de envolvi das no processo.

Palavras-chave Prescrição de Medicamentos; Serviços Farmacêuticos; Uso de Medicamentos; Informação sobre Medicamentos; Relações Profissional-Paciente 
Introdução

Os medicamentos constituem atual mente ferramentas poderosas para mitigar o sofrimento humano. Produzem curas, prolongam a vida e retardam o surgimento de complicações associadas às doenças, facilitando o convívio entre o indivíduo e sua enfermidade. Além disso, é possível considerar o uso apropriado e inteligente dos medicamentos como tecnologia altamente custo-efetiva, uma vez que pode influenciar, de modo substantivo, a utilização do restante do cuidado médico (Avorn, 1995).

O medicamento, como arma terapêutica, apresenta dois gumes. Por um lado, seu emprego inadequado pode provocar doenças iatrogênicas. Por outro, enquanto os países desenvolvidos investem na descoberta de novas substâncias com melhores características intrínsecas para o combate de problemas já resolvidos, os países em desenvolvimento ainda apresentam problemas de saúde que emergem das péssimas condições de vida da população, diminuindo a efetividade dos tratamentos medicamentosos já existentes.

A falta de serviços básicos de saneamento - redes de água e esgoto - que sirvam às camadas mais carentes e mais submetidas às desigualdades sociais, pode provocar, por exemplo, a recrudescência de doenças simples, como as parasitoses. Neste caso, o tratamento medicamentoso já não garantiria, per se, a cura do paciente.

A timidez - ou, mesmo, inexistência - de políticas sanitárias bem estruturadas, não resulta, de modo geral, em ações de promoção e prevenção à saúde. Ao contrário, traduz-se em medicina antes curativa que preventiva. Um contexto desprovido de política coerente para o setor saúde, dificilmente abrigará meios e esforços voltados para o desenvolvimento de política de medicamentos. No entanto, os medicamentos assumem importância exagerada frente à hegemonia da medicina curativa e desempenham papéis simbólicos tanto no sistema de saúde como para os próprios consumidores. De ferramentas disponíveis para promover e manter a saúde, costumam envergar o ônus de, entre outros, representar a própria saúde (Lefèvre, 1991; Sevalho, 1992; Scott \& Ferner, 1994).

Não obstante, há grande heterogeneidade de demanda medicamentosa nos países em desenvolvimento, o que reflete, muitas vezes, os desníveis sócio-econômi cos e culturais prevalentes (Lee et al., 1993). A Organização Mundial de Saúde (OMS, 1993), examinando o conceito de atenção farmacêutica, cita o acesso aos medicamentos como um dos fatores ne- cessários para a prestação da assistência à saúde. Assim, o acesso aos medicamentos essenciais à saúde da população é o primeiro passo para o estabelecimento de uma política de medicamentos.

Isto posto, interessa-nos ressaltar os momentos que pressupõem o contato direto entre as franjas do sistema de saúde - representado pelos profissionais médicos e farmacêuticos que nele atuam - e o alvo dos cuidados de saúde, o paciente. É durante o contato com o médico que o paciente recebe (ou não) a prescrição de medicamento. Entretanto, o uso adequado dos medicamentos não depende apenas de uma prescrição de qualidade, mas é também fruto de dispensação responsável. A interação entre paciente/médico/farmacêutico possibilita a emergência de expectativas, demandas e troca de informações que terão conseqüência direta no resultado da terapêutica.

Trataremos, de modo resumido, do processo que se desencadeia a partir do indivíduo que, por sentir-se doente, procura atendimento médico, o qual, comumente, finaliza na prescrição e dispensação de medicamento. Deternos-emos, em particular, na questão da produção e disseminação de informações, aos profissionais de saúde, a respeito dos medicamentos, bem como na interação entre paciente/ prescritor/dispensador.

\section{Informação sobre medicamentos}

A produção e a transmissão de informações a respeito dos medicamentos não se traduz necessariamente em conhecimento. Saraceno (1993) aborda, por exemplo, a informação que é veiculada aos prescritores acerca dos psicofármacos e considera o conhecimento como “(...) um trabal ho ativo e crítico das informações; é um processo complexo deativação denexos (...)" (Saraceno, 1993:164). Este processo é dificultado ou é inexistente quando há apenas o fenômeno da divulgação de informações, sem que se efetue sua transformação em conhecimento. Para este autor, a ocorrência e a importância deste fenômeno encontram-se na dependência de quem seja o produtor e o receptor das informações, assim como da cumplicidade existente entre ambos. Pensamos poder estender este raciocínio aos demais grupos farmacológicos e à transmissão de informações não somente aos prescritores, mas também aos dispensadores e pacientes.

Um primeiro aspecto a ser abordado está relacionado à produção e difusão da informação acerca dos medicamentos, incluindo-se aí 
as diversas fontes de informação existentes e disponíveis. Um segundo, refere-se à troca de informações, in loco, entre paciente/ prescritor/ dispensador.

Produção e difusão de informações a respeito de medicamentos

É notória a rapidez do desenvolvimento científico que - no que concerne às novas substâncias terapêuticas - se traduz em inúmeras possibilidades de produção de novos medicamentos oriundos tanto de processos extrativos ou de síntese química como da biotecnologia. Esta produção vem acompanhada da produção maciça de informação não somente para as agências regulamentadoras, mas igualmente para os profissionais de saúde e para os consumidores de medicamentos. Isto sem falar nas informações que, de forma rotineira, bombardeiam estes mesmos profissionais e consumidores no que diz respeito aos medicamentos já existentes no mercado. Trabalhá-las ativa e criticamente, com o intuito de transformá-las em conhecimento, significa, muitas vezes, uma tarefa hercúlea.

O trabal ho complica-se proporcionalmente à velocidade da produção e difusão de informações. No cruzamento de informações contraditórias, os profissionais - tal qual Sísifo carregam monte acima a pedra que rolará imediatamente após ter chegado ao topo.

Ainda que estejamos na era da informática - o que possibilita, na prática, acesso on lineà informação - , a maioria dos países em desenvolvimento está longe de utilizá-la de forma ampla e sistemática. Apenas poucos deleitamse por possuírem algum acesso à informatização e às informações originadas em fontes produtoras qualitativamente respeitáveis.

Este último aspecto é de fundamental importância, e o tipo de informação veiculada é justamente uma das questões que hoje se coloca. Recentemente têm aumentado não somente as informações, mas também a venda de medicamentos através de telefone e, mesmo, da Internet, atribuindo ao medicamento um lugar de mero bem de consumo e não de instrumento terapêutico.

As informações produzidas e difundidas aos profissionais e consumidores nem sempre são isentas, tornando necessária uma atitude crítica frente a elas. Os profissionais de saúde, prescritores e dispensadores têm sob sua responsabilidade, direta ou indiretamente, a saúde do paciente. É, portanto, essencial que busquem sempre o acesso a fontes fidedignas e atualizadas quanto aos medicamentos (Elanjian et al., 1993). Ainda mais que certas fontes de informação tentam transformar estes profissionais em "consumidores acríticos" de medicamentos (Temporão, 1986).

As fontes de informação produzidas e difundidas a respeito de medicamentos são de variados matizes, podendo ser resumidos da seguinte maneira:

a) Literatura científica publicada em revistas científicas independentes - este tipo de literatura pode ser exemplificado pelos periódicos internacionalmente reconhecidos. Ela é mais fidedigna, uma vez que há seleção mais cuidadosa e exigente quanto à qualidade dos artigos publicados. Esses periódicos encontram-se indexados e podem ser exemplificados, dentre outros, por British Medical Journal, New England Journal of Medicine, Lancet, Journal of the American Medical Association, American Journal of Health-System Pharmacy, Journal of the American Pharmaceutical Association;

b) Literatura publicada em outras revistas constituem as revistas não indexadas, algumas delas financiadas, ao menos em parte, pela indústria farmacêutica. Esta fonte geralmente só é conhecida no local de sua produção, não tendo expressão fora do país. A seleção dos artigos a serem publicados costuma ser mais complacente do que no caso anterior e pode ser mais freqüente a publicação de pesquisas que contenham problemas metodológicos e/ ou que contenham informações enviesadas e comprometidas:

c) Fontes de informação de cunho formativo produzida/ existente em locais de graduação/ pós-graduação - usualmente constituem material apostilado e resumido, distribuídos e lidos durante a formação médica efarmacêutica;

d) Fontes de informação oficiais, produzidas por organizações internacionais, como a Organização Mundial de Saúde (OMS), Organização Pan-americana de Saúde (OPAS) e United Nations Children's Emergency Fund (UNICEF), dentre outras - como exemplo, citamos: WHO Drug Information (WHO, s/d), Listas de Medicamentos Essenciais (MS, 1998), Informes Técnicos sobre Medicamentos e Lista Consolidada de Medicamentos Banidos (Department for Policy Coordination and Sustained Development, 1997).

e) Fontes de informação produzidas pela indústria farmacêutica - neste caso, as fontes são variadas e vão desde prospectos distribuídos aos profissionais de saúde por representantes até compilações de bulas medicamentosas publicadas anualmente, as quais, no Brasil, recebem os nomes de Dicionário das Especiali- 
dades Farmacêuticas (DEF) (Melo, 1999/2000) eP. R. Vade-mécum (Lépori, 1996). Outros exemplos importantes são as propagandas destinadas aos profissionais de saúde, veiculadas em periódicos correntes, outdoors, por intermédio do correio; "reuniões" e congressos promovidos e financiados pela indústria farmacêutica, que costumam focalizar determinado fármaco promocionado.

f) Livros-textos, contendo informações mais gerais - como os de Medicina e Farmácia - ou mais específicas, relativas à Farmacologia, Farmacologia Clínica, terapêutica e às reações adversas a medicamentos. Vários são os livros-textos existentes, nacionais ou estrangeiros, traduzidos ou não. Internacionalmente reconhecidos são, por exemplo: Goodman \& Gilman's: The Pharmacological Basis of Therapeutics (Gilman et al., 1993), Remington's Pharmaceutical Practice(Gennaro, 1990), AMA Drug Evaluation (AMA, 1996), Myeler's Side Effects of Drugs (Dukes, 1996), The Medical Letter Handbook of Drug Interactions (Rizack \& Hillman, 1998), The Handbook of Injectable Drugs (Trissel, 1996), Oxford Textbook of Clinical Pharmacology (Grahame-Smith \& Aronson, 1992). Um dos problemas nesse tipo de publicação é a dificuldade de acesso a edições recentes seja pelo preço de aquisição seja pela existência de traduções apenas das edições mais antigas. Cabe citar a existência de compêndios bastante utilizados, que contêm informações atualizadas regularmente a respeito da maior parte dos fármacos existentes. Os mais conhecidos deles são o Martindale's, The Extra Pharmacopoeia (Reynolds, 1993) e o British National Formulary (Joint Formulary Committee of the British Medical Association \& The Royal Pharmaceutical Society of Great Britain, 1997).

g) Informações trocadas entre os profissionais - podem ocorrer durante a prática diária; em reuniões promovidas nos locais de trabaIho, como centro de estudos, discussão de casos, Congressos, reuniões científicas e cursos realizados nas sociedades ou associações profissionais. Há que chamar a atenção para a rede existente, no Brasil, de Centros de Informações sobre Medicamentos (CIMs), que funcionam com base em consultas dos profissionais de saúde e dos consumidores feitas a órgãos profissionais ou universidades.

h) Farmacopéias - grande número de países tem formado uma comissão elaboradora de sua farmacopéia. A farmacopéia contém monografias de substâncias medicamentosas, listas de soluções reagentes, soluções indicadoras e ensaios microbiológicos, químicos, físicos e físico-químicos utilizados no controle da qua- lidade dos medicamentos. Como exemplos citamos TheUnited States Pharmacopoeia (USPC, 1999), a British Pharmacopoeia (British Pharmacopoeia Commission, 1999) e a Farmacopéia Brasileira (Comissão Permanente de Revisão da Farmacopéia Brasileira, 1988).

i) Informações disponibilizadas através do computador - vão desde os anúncios citados anteriormente até bases de dados que contêm informações relativas a medicamentos, como MED-LINE, DRUG-LINE, COCHRANE LIBRARY, MICROMEDEX. Atualmente, por intermédio da INTERNET, pode-se ter acesso a periódicos internacionais como New England Journal of Medicine, Lancet, British Medical Journal, a publicações da OMS e a guidelines para situações específicas. Encontra-se, igualmente, disponível o acesso a agências regulamentadoras de outros Países como, por exemplo, o Food and Drug Administration (FDA) dos Estados Unidos da América.

Os prescritores necessitam ter acesso a informações acerca da relevância clínica dos medicamentos, em particular, quanto às indicações, efeitos adversos, bem como ao custo comparado com alternativas disponíveis (Bero \& Rennie, 1996). As informações menos distorcidas são aquelas baseadas em dados resultantes de ensaios clínicos controlados bem desenhados e de estudos de farmacovigilância e de utilização dos medicamentos (Saraceno, 1993).

No entanto, cabe ao receptor destas informações saber selecionar os estudos menos enviesados, uma vez que alguns resultados visam objetivos mais comerciais do que propriamente científicos. Bero \& Rennie (1996) referem que, entre 1980 e 1986, 61\% dos ensaios clínicos foram realizados nesta condição e que, com freqüência, os estudos de custo-efetividade, nos Estados Unidos, são elaborados pelo departamento de marketing - não o de pesquisa - das indústrias farmacêuticas. Em recente publicação referida à controvérsia quanto aos antagonistas do canal de cálcio, Stelfox et al. (1998) concluem que, na literatura de língua inglesa, as posições publicamente assumidas, entre março de 1995 e setembro de 1996, contra ou a favor destas substâncias estavam fortemente associadas à existência ou não de relações de financiamento dos autores com a indústria farmacêutica.

A velocidade na adoção de "inovações" varia entre os profissionais médicos, sendo alguns mais rápi dos e outros mais cuidadosos na incorporação, a sua prática, das novidades lançadas no mercado (Felch \& Scanlon, 1997). Diversificada é igualmente a maneira como os profissionais recebem e consideram as infor- 
mações recebidas. Neste sentido, os médicos são indivíduos complexos que, no processo de construção de sua prática, ganham novas informações, testando-as no dia-a-dia e comparando-as com o conhecimento anteriormente e internamente consolidado (Felch $\&$ Scanlon, 1997).

O prescritor tende a ponderar que as fontes de informação científicas de boa qualidade são aquelas que mais influenciam sua decisão no momento da escolha entre prescrever/não prescrever e qual o medicamento a ser prescrito. Eles não percebem, por vezes, que são levados à prescrição de medicamentos cuja eficácia e segurança não se encontram bem estabelecidas (Pepe \& Travassos, 1995). Alguns estudos referem que o contato com colegas, cursos, leitura de periódicos estão entre as principais fontes de informação utilizadas pelos médicos (Felch \& Scanlon, 1997). Outros autores, apesar de obterem achados semelhantes, supõem que as fontes de informação oriundas da indústria farmacêutica exercem considerável influência, ao menos subjetivamente, na prescrição dos médicos (Batellino, 1985; Orlowski \& Wateska, 1992).

De qualquer maneira, apenas o conhecimento acerca dos medicamentos não é suficiente para a terapêutica alcançar seu melhor resultado. Ele vai estar relacionado, também, ao processo que se desencadeia a partir do momento em que um paciente começa a falar de seus males e da forma como se comunicarão paciente, prescritor e dispensador. Chegamos, assim, ao nosso segundo tema de interesse.

Troca de informações e interação entre paciente/prescritor/dispensador

\section{Prescritor/dispensador}

A integração entre prescritores e dispensadores permite, através da combinação de conhecimentos especializados e complementares, o alcance de resultados eficientes, beneficiando o paciente (Rupp et al., 1992).

A priorização do papel da equipe multidisciplinar em detrimento daqueles papéis corporativos - tendência anunciada pela visão compartimentalizada das práticas médica e farmacêutica - tornou fato o reconhecimento do trabalho do farmacêutico e de outros profissionais de saúde, possibilitando o desenvolvimento de atividades comuns e absolutamente essenciais, em ponto nevrálgico: o medicamento (Bonal, 1979; ASHP, 1996; Avorn, 1997).
O envolvimento atento do farmacêutico na dispensação, bem como sua função educativa, é de valia no suprimento de informações ao médico assistente ou ao corpo clínico da instituição. Não deveria ser incomum que o prescritor, quando sem acesso a informações completas e atualizadas acerca de fármacos, recorresse ao farmacêutico, que, por sua vez, pudesse solicitar também informações e esclarecimentos do médico em sua área de competência.

No entanto, o relacionamento entre médicos e farmacêuticos, no terreno da prescrição/ dispensação, tem tido seus percalços. Alguns autores (Kapil, 1988; Cowen, 1992) relatam fatos do passado, quando os farmacêuticos eram percebidos pelos médicos como verdadeiros "usurpadores" no campo da dispensação, que era entendida pela classe médica como atividade exclusiva. Mesmo hoje em dia, na Inglaterra, por exemplo, ainda se discutem as delimitações profissionais: médicos dispensando e farmacêuticos clinicando são práticas que convivem não sem atritos (RPSGB, 1997).

As responsabilidades do farmacêutico frente às prescrições médicas têm merecido reflexões. Este profissional encontra-se na interface entre a distribuição de fármacos e o seu uso, podendo ser considerado como peça-chave na garantia da qualidade do cuidado médico (Rupp et al., 1992). Ele representa uma das últimas oportunidades de, ainda dentro do sistema de saúde, identificar, corrigir ou reduzir possíveis riscos associados à terapêutica. Suas responsabilidades, no momento da dispensação, são múltiplas. Envolvem questões de cunho legal, técnico e clínico. No momento que antecede o aviamento da receita/ prescrição, o farmacêutico pode examiná-la atentamente, cruzando estas informações com dados da história clínica do paciente. É imprescindível o total entendimento das informações constantes na prescrição (Brown, 1997).

Os riscos associados à terapêutica podem ser minimizados pelo investimento na qualidade da prescrição e dispensação (Bates et al., 1997). Um aspecto associado ao incremento dessa qualidade seria a prevenção das reações adversas e da interação medicamentosa, comum em situações quando mais de um fármaco é utilizado. Por outro lado, completando o cenário, o farmacêutico pode ter acesso a relevantes informações quanto à história do paciente (múltiplos prescritores) e a regimes terapêuticos paralelos e/ ou concomitantes (polifarmácia). 
Paciente/prescritor

A prescrição é, na maioria das vezes, feita pelo profissional médico. Ela é o resultado de uma série complexa de decisões que este profissional vai tomando durante a consulta, após entrar em contato com o paciente. A importância do prescritor reside no fato de ser ele o responsável pela indicação de um medicamento com base na interpretação que faz daquilo que o paciente lhe diz. A importância do paciente reside em ser ele quem vai descrever o que está sentindo e quem vai aderir, ou não, à indicação médica. Ambos carregam consigo vivências e expectativas que Ihes permitem tomar decisões quanto à prescrição e uso de determinado medicamento; neste contato, muitos são os fatores que podem influenciá-los.

O prescritor pode ser influenciado por características que lhe são próprias, ou por fatores externos a ele, tal como: local de atendimento, agências regulamentadoras, propaganda, comuni dade acadêmica e interesses econômicos (Higginbotham \& Streiner, 1991). Certas características relativas ao paciente são, da mesma forma, importantes para a tomada de decisão médica: as expectativas e demandas dos pacientes, sua família, seus empregadores; a atitude do paciente frente à saúde; suas características físicas como peso, idade, sua sensibilidade aos medicamentos; sua condição econômica e inserção no mercado de trabalho (Pepe \& Travassos, 1995).

Por sua vez, os pacientes buscam os médicos cuja prática vai ao encontro de suas aspirações e, de certa forma, pode tanto avaliar o médico em função do remédio como vice-versa. O medicamento pode significar que o médico sabe a respeito de sua doença e que está zelando por ela.

Um levantamento realizado, em 1993, incluindo 124 médicos (general istas, cirurgiões) e seus pacientes, em Oregon e Colorado (Estados Unidos), sugere que os pacientes dão valor ao interesse, à atenção, aos conselhos e à informação prestados pelo médico durante a consulta. Para estes autores, um encontro acolhedor faz o paciente sentir-se como pessoa real e não apenas uma doença, aos olhos do médico (Levinson et al., 1997).

Outro aspecto merece aqui consideração e diz respeito à diferença que pode existir entre o prescritor e o paciente na avaliação do medicamento. Os pacientes não avaliam a efetividade do medicamento somente em termos de seu efeito na doença propriamente dita, ou diminuição dos sintomas, mas também o quanto o tratamento Ihes permite lidar com outras situações de sua vida social (Higginbotham \&
Streiner, 1991). O paciente pode perceber como melhor ou pior alguns atributos medicamentosos, culturalmente significativos, classificando-os por conta própria: pela coloração, pela via de administração, pelo fato de ser comprado ou dado etc. (Higginbotham \& Streiner, 1991; Pepe \& Travassos, 1995).

Enfim, o medicamento pode simbolizar a saúde ou a cura, objetivos da relação terapêutica, reproduzir, de certa forma, o poder do médico sobre o paciente (Lefèvre, 1991). A prescrição pode ser vista como maneira satisfatória, para paciente e prescritor, de terminar uma consulta, e o contato entre prescritor/ paciente é de fundamental importância para o resultado final do tratamento (Higginbotham \& Streiner, 1991). É a partir deste encontro que o médico vai decidir a necessi dade da prescrição e escoIher, caso positivo, o melhor medicamento para cada paciente; este vai decidir se seguirá as recomendações feitas de forma integral, parcial ou se não aderirá ao tratamento proposto.

\section{Paciente/dispensador}

A dispensação não configura apenas a ocasião em que determinada receita ou prescrição é aviada. Não é troca de mercadorias por receita médica. Tão, ou mais, importante que o medicamento recebido pelo paciente, é a informação envolvida.

O fluxo da informação, iniciado pela consulta médica, tem continuidade no recinto da farmácia, onde o paciente vai ser esclarecido a respeito dos itens da prescrição médica, dose, posologia, cuidados especiais na reconstituição e/ ou armazenamento, considerações ao tomar o medicamento, possíveis interações, efeitos adversos etc. (Newton et al., 1996). Esta função informativa e educativa da dispensação tornaa peça chave na cadeia da assistência à saúde. Este aconsel hamento, no ato da dispensação, dá oportunidade à criação de novo vínculo assistencial (Hepler \& Strand, 1990).

Tradicional campo do farmacêutico, a dispensação não é julgada pela sociedade - constituída por potenciais pacientes - como atividade de alto valor (Arancíbia, 1990). Habitualmente, o paciente tem dificuldade em associar o recinto "farmácia" como um dos locais privilegiados para a troca de informações. É sugerido que os pacientes sequer esperam contato com o farmacêutico. No entanto, a dispensação pode tornar-se encontro profícuo paciente entre e dispensador (Johnson et al., 1996; Nau et al., 1997).

Por seu lado, com freqüência, o paciente coloca - em especial, nas farmácias comunitárias - o dispensador (farmacêutico ou balco- 
nista) no papel de prescritor. Ou seja, é possível que, nestas situações, o fato de entrar em contato com o dispensador possa parecer prescindível o papel do prescritor, como se fossem atividades excludentes e não complementares. Cabe ao dispensador eximir-se desta prática, referenciando o indivíduo ao sistema de saúde (Schommer \& Wiederholt, 1995).

O exercício da dispensação direta ou supervisionada pelo farmacêutico, além de favorecer a veiculação de informações ao paciente, despertará seu interesse para as atividades do farmacêutico (Levinson et al., 1997). É desejável, pois, sempre que possível, oferecer assistência direta, seja em situações ambulatoriais, hospitalares e comunitárias, de modo a, por meio da boa prática, demonstrar sua importância (Nau et al., 1997), especialmente como integradora das diversas informações recebidas pelo paciente desde o início de sua chegada ao serviço de saúde.

\section{Considerações finais}

A prescrição de medicamento simboliza importante dimensão do processo terapêutico ea "transformação" desta prescrição em comprimidos, suspensões etc., pelo farmacêutico, prolonga este encontro por dias ou até mesmo meses após a sua realização (Lefèvre, 1991; Avorn, 1995). O paciente chega a este encontro trazendo consigo sua singularidade, fruto de seus sa-

\section{Referências}

AMA (American Medical Association), 1996. AMA Drug Evaluation. 6th Ed. Philadelphia: W. B. Saunders.

ASHP (American Society of Hospital Pharmacists), 1996. ASHP guidelines on medication-use evaluation. American Journal of Health-System Pharmacy, 53:1953-1955.

ARANCIBIA, A., 1990. La crisis de identidad professional del farmacéutico en América Latina. Pharmaklinik, 4:11-25.

AVORN, J., 1995. The prescription as a final common pathway. International Journal of Technology Assessment in Health Care, 11:348-390.

AVORN, J., 1997. Putting adverse drug events into perspective. JAMA, 227:341-342.

BATELLINO, L. J., 1985. La información farmacológica y la actividad médica. Cuadernos Médico Sociales (Rosario), 34:25-43.

BATES, D. W.; SPELL, N.; CULLEN, D. J.; BURDICK, E.; LAIRD, N.; PETERSEN, L. A.; SMALL, S. D.; SWEITZER, B. J. \& LEAPE, L. L., 1997. The costs of adverse drug events in hospitalized patients. JAMA, 277:307-311. beres, crenças e experiências a respeito do processo saúde/ doença. O mesmo dá-se com os profissionais de saúde, prescritores e dispensadores. Algumas influências são decisivas neste processo e se traduzem não somente nas chamadas variações da prática médica como também em variações das respostas que o paciente dá a este encontro. Assim, o prescritor pode ou não prescrever o melhor medicamento para aquele paciente, o dispensador pode ou não dispensar o medicamento prescrito da melhor maneira e o paciente pode ou não aderir ao tratamento.

Na melhor das hipóteses, se tudo correr a contento, o medicamento realmente servirá para mitigar o sofrimento do indivíduo. Pode ser também que este objetivo não seja alcançado e o indivíduo continue em busca de outras opções. Na pior das hipóteses, é possível que se traduza em mais e/ ou maior sofrimento e, neste caso, o sistema de saúde ao invés de tratar as doenças, acaba por produzi-las.

Enfim, há uma multiplicidade de fatores a interferir no resultado de uma prescrição, desde a decisão médica relativa à prescrição até a ingestão de cada dose medicamentosa pelo paciente e os efeitos daí advindos. Quanto meIhor é o processo de produção de conhecimento a partir das informações recebidas no que concerne aos medicamentos e quanto melhor a interação entre prescritor, dispensador e paciente, mais próximo se está de alcançar um melhor resultado.

BERO, L. \& RENNIE, D., 1996. Influences on the quality of published drug studies. International Journal of Technology Assessment in Health Care, 12: 209-237.

BONAL, J., 1979. Farmacia clínica: Un instrumento sanitario de control de calidad. Medicina de Posgrado, 1:298-302.

BRITISH PHARMACOPOEIA COMMISSION, 1999. British Pharmacopoeia. London: The Stationery Office.

BROWN, T. A., 1997. Reviewing and dispensing prescription and medication orders. In: Comprehensive Pharmacy Review (L. Shargel, A. H. Mutnick, P. F. Souney, L. N. Swanson \& L. H. Block, eds.), pp. 428-435, Baltimore: Williams \& Wilkins.

COMISSÃO PERM ANENTE DE REVISÃO DA FARMACOPÉIA BRSILEIRA, 1988. Farmacopéia Brasileira. São Paulo: Atheneu.

COWEN, D. L., 1992. Changing relationship between pharmacists and physicians. American Journal of Hospital Pharmacy, 49:2715-2721.

DEPARTMENT FOR POLICY COORDINATION AND SUSTAINED DEVELOPMENT, 1997. Consolidated 
List of Products Whose Consumption and/or Sale Have Been Banned, Withdraw, Severely Restricted or Not Approved by Governments. New York: Department for Policy Coordination and Sustained Development.

DUKES, M. N. G., 1996. Myeler's Side Effects of Drugs. 13th Ed. Amsterdam: Elsevier Science Ltd.

ELANJIAN, S.; GORA, M. L. \& SYMES, L. R., 1993. Methods used by pharmacy departments to identify drug interactions. American Journal of Hospital Pharmacy, 50:2546-2549.

FELCH, W. C. \& SCANLON, D. M., 1997. Bridging the gap between research and practice: The role of continuing medical education. JAMA, 277:155-156.

GENNARO, A. R., 1990. Remington's Pharmaceutical Sciences. 18th Ed. Easton: Mack.

GILMAN, A. G.; RALL, T. W.; NIES, A. S. \& TAYLOR, P., 1993. Goodman \& Gilman's The Pharmacological Basis of Therapeutics. 8th Ed. New York: McGrawHill.

GRAHAME-SMITH, D. G. \& ARONSON, J. K., 1992. Oxford Textbook of Clinical Pharmacology. Oxford: Oxford University Press.

HEPLER, C. D. \& STRAND, L. M., 1990. Opportunities and responsibilities in pharmaceutical care. American Journal of Hospital Pharmacy, 47:533-543.

HIGGINBOTHAM, N. \& STREINER, D. L., 1991. The social science contribution to pharmacoepidemiology. Journal of Clinical Epidemiology, 44(Sup. 2): $73 \mathrm{~S}-82 \mathrm{~S}$

JOHNSON, K. B.; BUTTA, J. K.; DONOHUE, P. K.; GLENN, D. J. \& HOLTZMAN, N. A., 1996. Discharging patients with prescriptions instead of medications: Sequelae in a teaching hospital. $\mathrm{Pe}$ diatrics, 97:481-485.

JOINT FORM ULARY COMMITTEE OF THE BRITISH MEDICAL ASSOCIATION \& THE ROYAL PHARMACEUTICAL SOCIETY OF GREAT BRITAIN, 1997. British National Formulary. Wallingford: The Pharmaceutical Press.

KAPIL, I., 1988. Doctors dispensing medications Contemporary India and 19th century England. Social Science and Medicine, 26:691-698.

LEE, D.; BALASUBRAM ANIAM, K. \& ALI, H. M., 1993. Drug utilization studies: Their transferability between industrialized and developing countries. In: Drug Utilization Studies. Methods and Uses (M. N. G. Dukes, ed.), pp. 193-218, Copenhagen: WHO Regional Publications, World Health Organization.

LEFÈVRE, F., 1991. O Medicamento como Mercadoria Simbólica. São Paulo: Cortez.

LÉPORI, L. R., 1996. P. R. Vade-mécum. 2a Ed. São Paulo: Soriak.

LEVINSON, W.; ROTER, D. L.; MULLOOLY, J. P.; DULL, V. T. \& FRANKEL, R. M., 1997. Physician-patient communication. JAMA, 277:553-559.

MELO, J. M. S., 1999/2000. Dicionário das Especialidades Farmacêuticas. São Paulo: Epuc.

MS (Ministério da Saúde), 1998. Listas de Medicamentos Essenciais. «ttp:// www.saude.gov.br/rename. htm>

NAU, D. P.; RIED, D. \& LIPOWSKI, E., 1997. What makes patients think that their pharmacists' services are of value? Journal of the American Pharmaceutical Association, 37:91-98.
NEWTON, G. D.; PRAY, W. S. \& POPOVICH, N. G., 1996. New OTC drugs and devices: A selected review. Journal of the American Pharmaceutical Association, 36:108-112.

OMS (Organización Mundial de la Salud), 1993. El Papel del Farmacéutico en el Sistema de Atención de Salud. Informe de la Reunión de la OMS, Tokio, Japón, 31 de agosto al 3 de septiembre de 1993. Washington, D.C.: Organización Panamericana de la Salud.

ORLOWSKI, J. P. \& WATESKA, L., 1992. The effects of pharmaceutical firm enticements on physician prescribing patterns: There's no such thing as a free lunch. Chest, 102:266-269.

PEPE, V. L. E. \& TRAVASSOS, C. M., 1995. A Prescrição Médica. Rio de Janeiro: Instituto de Medicina Social, Universidade do Estado do Rio de Janeiro.

REYNOLDS, J. E. F., 1993. Martindale's The Extra Pharmacopoeia. 30th Ed. London: The Pharmaceutical Press.

RIZACK, M. A. \& HILLMAN, D. M., 1998. The Medical Letter Handbook of Adverse Drug Interactions. London: The Medical Letter.

RPSGB (The Royal Pharmaceutical Society of Great Britain), 1997. Dispensing doctors' higher costs confirmed. Pharmaceutical Journal, 258:88.

RUPP, M. T.: DeYOUNG, M. \& SCHONDELMEYER, S W., 1992. Prescribing problems and pharmacist interventions in community practice. Medical Care, 30:926-940.

SARACENO, B., 1993. Questões abertas em psicofarmacologia. In: Duzentos Anos de Psiquiatria (J. F. Silva Filho \&J. Russo, org.), pp.163-80, Rio de Janeiro: Editora Relume-Dumará/Universidade Federal do Rio de Janeiro.

SCHOM MER, J. C. \& WIEDERHOLT, J. B., 1995. A field investigation of participant and environment effects on pharmacist-patient communication in community pharmacies. Medical Care, 33: 567584.

SCOTT, D. K. \& FERNER, R. E., 1994. The strategy of desire and rational prescribing. British Journal of Clinical Pharmacology, 37:217-219.

SEVALHO, G., 1992. Permanências culturais e uso do medicamento alopático moderno. Dissertação de Mestrado, Rio de Janeiro: Escola Nacional de Saúde Pública, Fundação Oswaldo Cruz.

STELFOX, H. T.; CHUA, G.; O'ROURKE, K. \& DETSKY, A. S., 1998. Conflict of interest in the debate over calcium-channel antagonists. New England Journal of Medicine, 338:101-106.

TEM PORÃO, J. G., 1986. A Propaganda de Medicamentos e o Mito da Saúde. Rio de Janeiro: Graal.

TRISSEL, L. A., 1996. The Handbook of Injectable Drugs. Bethesda: American Society of Hospital Pharmacists.

USPC (United States Pharmacopeial Convention), 1999. The United States Pharmacopeia. The National Formulary. Rockville: USPC.

WHO (World Health Organization), s/d. WHO Drug Information. Geneva: WHO. 